PROCEEDINGS OF THE

AMERICAN MATHEMATICAL SOCIETY

Volume 137, Number 4, April 2009, Pages 1245-1254

S 0002-9939(08)09634-2

Article electronically published on October 20, 2008

\title{
GROUP GRADINGS ON SIMPLE LIE ALGEBRAS IN POSITIVE CHARACTERISTIC
}

\author{
YURI BAHTURIN, MIKHAIL KOCHETOV, AND SUSAN MONTGOMERY
}

(Communicated by Birge Huisgen-Zimmermann)

\begin{abstract}
In this paper we describe all gradings by a finite abelian group $G$ on the following Lie algebras over an algebraically closed field $F$ of characteristic $p \neq 2: \mathfrak{s l}_{n}(F)(n$ not divisible by $p), \mathfrak{s o}_{n}(F)(n \geq 5, n \neq 8)$ and $\mathfrak{s p}_{n}(F)$ ( $n \geq 6, n$ even).
\end{abstract}

\section{INTRODUCTION}

We are interested in gradings on finite-dimensional simple Lie algebras over an algebraically closed field $F$. If a simple Lie algebra $L$ is graded by a group $G$, then the support of the $G$-grading on $L, \operatorname{Supp} L:=\left\{g \in G \mid L_{g} \neq 0\right\}$, generates an abelian subgroup of $G$ [6, Lemma 2.1]. Thus it is sufficient to consider the case when $G$ is abelian. If $L$ is finite-dimensional, one can also assume that $G$ is finitely generated. Gradings by the groups $G=\mathbb{Z}^{n}$ correspond to actions by tori. They have been extensively studied and find numerous applications (see e.g. [11]). We will restrict ourselves to the case of finite groups. Gradings by finite groups arise in the study of generalized symmetric spaces in differential geometry (see e.g. [12] and many more references in [1]), in the theory of Kac-Moody algebras 13, and also in the classification of infinite-dimensional simple Lie algebras endowed with a finite grading by a torsion-free group [15]. For some applications it is desirable to know all possible gradings on a given Lie algebra - e.g. in the context of symmetric spaces [1] and for the classification of simple Lie coloralgebras via the coloration-discoloration process.

In the case char $F=0$, all gradings on the classical simple Lie algebras (except of type $D_{4}$ ) have been described in 3,6 , 4. Here we will focus on the case char $F=$ $p>0$. We will assume that $p \neq 2$.

Our main results are Theorems 5.1 and 5.5 where we prove, respectively, that any grading on $\mathfrak{s l}_{n}(F), n \geq 2$, in characteristic $p \neq 2$, with $p \nmid n$, and any grading on $\mathfrak{s o}_{n}(F), n \geq 5, n \neq 8$, and on $\mathfrak{s p}_{n}(F), n$ even, $n \geq 6$, in characteristic $p \neq 2$,

Received by the editors July 5, 2007, and, in revised form, February 8, 2008, and, April 21, 2008.

2000 Mathematics Subject Classification. Primary 16W10, 16W50, 17B50, 17B70.

The first author was partially supported by NSERC grant \# 227060-04 and by a URP grant, Memorial University of Newfoundland.

The second author was supported by a Start-up Grant, Memorial University of Newfoundland.

The third author was supported by NSF grant DMS 0401399.

(C)2008 American Mathematical Society 
is of the same kind as in characteristic 0 and thus may be completely described in terms of gradings on the full matrix algebra $M_{n}(F)$.

All gradings on the full matrix algebra $R=M_{n}(F)$ by an arbitrary group $G$ have been classified, up to conjugation by a nonsingular matrix, in [5]. Namely, there exist graded unital subalgebras $A \cong M_{k}(F)$ and $B \cong M_{l}(F)$ in $R$ such that $R=A \otimes B$ (thus $k l=n$ ), $A$ has a fine grading (i.e., $\operatorname{dim} A_{g} \leq 1$ for each $g \in G$ ), and $B$ has an elementary grading defined by an $l$-tuple $\left(g_{1}, \ldots, g_{l}\right)$ of elements of $G$ (i.e., $B_{g}=\operatorname{span}\left\{E_{i j} \mid g_{i}^{-1} g_{j}=g\right\}$ for each $g \in G$, where $\left\{E_{i j}\right\}$ is a basis of matrix units in $B$ ). If char $F \nmid k$, then $A$ decomposes as the tensor product of graded matrix algebras whose homogeneous components are spanned by the so-called generalized Pauli matrices. The support of a fine grading on $A$ is a subgroup in $G$ of order $k^{2}$ [5. Theorem 8]. In particular, when $\operatorname{char} F=p$ and $|G|$ is a power of $p$, then all $G$-gradings on $M_{n}(F)$ are elementary.

To deal with the case when $G$ is an abelian $p$-group, we use duality (recalled in Section (2) to translate the problem to the action of a certain divided power Hopf algebra $K$ on $R=M_{n}(F)$ with $p \nmid n$, considered as a Lie algebra, and prove in Section 4 that any such Hopf action is in fact an action on $R$ considered as an associative algebra, provided $1_{R}$ is $K$-invariant. It turns out that this result holds for any connected cocommutative Hopf algebra $K$, and it also extends to the other classical simple Lie algebras (see Theorem 4.1). The proof uses the techniques from the theory of formal groups, which is briefly recalled in Section 3 We then combine this case with the methods used in characteristic zero [6] to prove our main results in Section 5 for any finite abelian group $G$.

\section{Duality}

Let $H$ be a Hopf algebra over $F$. We will use the summation notation for comultiplication: $\Delta(h)=\sum h_{1} \otimes h_{2}$, for any $h \in H$. If $G$ is a group, then the group algebra $F G$ of $G$ over $F$ can be regarded as a Hopf algebra, with comultiplication $\Delta(g)=g \otimes g$, counit $\varepsilon(g)=1$, and antipode $S(g)=g^{-1}$, for all $g \in G$.

Let $A$ be an algebra over $F$, not necessarily associative. It is well-known that a $G$-grading on $A$ is equivalent to the structure of a right $F G$-comodule algebra (see for example [14, 1.6.7, 4.1.7] for the associative case). Recall that $A$ is an $H$ comodule algebra if there is a homomorphism of algebras $\rho: A \rightarrow A \otimes H$, written as $\rho(a)=\sum a_{0} \otimes a_{1}$ where $a_{0} \in A$ and $a_{1} \in H$, such that $(\rho \otimes i d) \rho=(i d \otimes \Delta) \rho$. When $A$ is unital associative, one also requires that $1_{A} \in A^{c o H}$, the $H$-coinvariants of $A$ (i.e., $\rho\left(1_{A}\right)=1_{A} \otimes 1_{H}$ ).

In the case of a $G$-graded algebra $A=\bigoplus_{g \in G} A_{g}, \rho$ is defined on a homogeneous element $a \in A_{g}$ by $\rho(a)=a \otimes g$. Conversely, given a suitable homomorphism $\rho: A \rightarrow A \otimes F G$, one can define a $G$-grading on $A$ by setting $A_{g}=\{a \in A \mid \rho(a)=$ $a \otimes g\}$, for any $g \in G$. Clearly $A_{1}$ is the set of $F G$-coinvariants.

If $H$ is any finite-dimensional Hopf algebra $H$ with dual Hopf algebra $K=H^{*}$, then $A$ is a right $H$-comodule algebra $\Longleftrightarrow$ it is a left $K$-module algebra; i.e., $k \cdot(a b)=\sum\left(k_{1} \cdot a\right)\left(k_{2} \cdot b\right)$ for all $k \in K, a, b \in A$ (see [14]). The action of $K$ on $A$ is given by $f \cdot a=(i d \otimes f) \rho(a)$. It follows from the above equivalence that $B$ is a subcomodule of a right $H$-comodule $A$ if and only if $B$ is a left $K$-submodule of $A$. Moreover, $1_{A}$ is an $H$-coinvariant $\Longleftrightarrow$ it is a $K$-invariant. 
Remark 2.1. In order to show that $A$ is a $K$-module algebra, it suffices to show that $A$ is a $K$-module and that the equation $k \cdot(a b)=\sum\left(k_{1} \cdot a\right)\left(k_{2} \cdot b\right)$ holds for all $k$ in a set of algebra generators of $K$ (and if $1_{A} \in A$, then $1 \in A^{K}$ ).

Now $H=H_{1} \otimes H_{2}$ implies $H^{*} \cong H_{1}^{*} \otimes H_{2}^{*}$. By the remark, to show that $A$ is an $H^{*}$-module algebra, it suffices to show it is a module algebra for $H_{1}^{*}$ and $H_{2}^{*}$.

We now specialize to the case of a graded algebra $A$ and $H=F G$. The dual Hopf algebra is $K=(F G)^{*}$, and the action of $K$ on $A$ is given by $f \cdot a=f(g) a$ for any $a \in A_{g}, g \in G$. Since $F G$ is cocommutative, $K$ is commutative.

From now on, $G$ is a finite abelian group and $F$ is an algebraically closed field of characteristic $p>0$. Then $K$ is also cocommutative. We may write $G=$ $G_{0} \times G_{1}$, where $G_{0}$ is of order relatively prime to $p$ and $G_{1}$ is a $p$-group. Then $H=F G_{0} \otimes F G_{1}=H_{0} \otimes H_{1}$, and thus $K=\left(F G_{0}\right)^{*} \otimes\left(F G_{1}\right)^{*}=K_{0} \otimes K_{1}$. It suffices to describe the dual Hopf algebras for the two cases $K_{0}$ and $K_{1}$.

1) First, assume that $|G|$ is relatively prime to $p$. In this case, since $F$ is algebraically closed, it is well-known that $K=(F G)^{*}=F \widehat{G}$, where $\widehat{G}$ is the group of multiplicative characters of $G$. Moreover, any $\chi \in \widehat{G}$ is a group-like element of $K$. Thus $G$-gradings on an algebra $A$ are equivalent to actions of $\widehat{G}$ on $A$ as automorphisms. Such actions by $\widehat{G}$ were used for gradings in characteristic 0 .

2) Now assume that $G$ is a $p$-group; this case is quite different. Then $K$ is a connected Hopf algebra in the sense that the coradical of $K$ is spanned by $1_{K}$. The theory of connected cocommutative Hopf algebras is closely related to the theory of formal groups. In the following section we recall the facts that we will need in the proof of Theorem 4.1 .

\section{Formal Groups}

Let $K$ be a connected cocommutative bialgebra. It is well-known that any such $K$ has an antipode and thus is a Hopf algebra [10, II, $\S 2$, No. 8]. We denote by $P(K)$ the Lie algebra of primitive elements of $K: P(K)=\{k \in K \mid \Delta k=k \otimes 1+1 \otimes k\}$. It is well-known that in characteristic zero $K$ is isomorphic to $U(P(K))$. In the case of a perfect field of characteristic $p>0$ and assuming $\operatorname{dim} P(K)=d<\infty$, one can construct a basis of "divided powers" $\left\{z^{(\alpha)}\right\}$ in $K$ that has the following properties [10, II, $\S 2$, No. 6 and No. 9]:

A) $\alpha=\left(\alpha_{1}, \ldots, \alpha_{d}\right)$ runs over the set

$$
L(d, \bar{r})=\left\{\alpha \in \mathbb{Z}^{d} \mid 0 \leq \alpha_{i}<p^{r_{i}+1}, i=1, \ldots, d\right\},
$$

where $\bar{r}=\left(r_{1}, \ldots, r_{d}\right), r_{i} \in \mathbb{Z}_{+} \cup\{\infty\}, \mathbb{Z}_{+}=\{0,1,2, \ldots\}$, with the convention that $\alpha_{i}$ is not bounded above if $r_{i}=\infty$.

B) For any $\alpha \in L(d, \bar{r})$,

$$
\Delta z^{(\alpha)}=\sum_{\beta+\gamma=\alpha} z^{(\beta)} \otimes z^{(\gamma)} .
$$

C) Let $\varepsilon_{i} \in L(d, \bar{r})$ have 1 in the $i$-th position and 0 elsewhere. Then $z^{\left(\varepsilon_{i}\right)}$, $i=1, \ldots, d$, form a basis of $P(K)$.

As a consequence, one obtains that $z^{(0)}=1_{K}$ and $\varepsilon_{K}\left(z^{(\alpha)}\right)=\delta_{\alpha, 0}$ (the Kronecker symbol). It follows from A) and B) that the dual algebra $K^{*}$ is isomorphic to the quotient of the algebra of formal power series $F\left[\left[t_{1}, \ldots, t_{d}\right]\right] /\left(t_{1}^{p^{r_{1}+1}}, \ldots, t_{d}^{p^{r_{d}+1}}\right)$, with no relation for $t_{i}$ if $r_{i}=\infty$. Namely, a power series $f=\sum_{\alpha} \lambda_{\alpha} \bar{t}^{\alpha}$ (where $\bar{t}^{\alpha}$ 
stands for $\left.t_{1}^{\alpha_{1}} \cdots t_{d}^{\alpha_{d}}\right)$ defines a functional on $K$ by $\left\langle f, z^{(\alpha)}\right\rangle=\lambda_{\alpha}$; in particular, $\left\langle\bar{t}^{\alpha}, z^{(\beta)}\right\rangle=\delta_{\alpha, \beta}$. The bialgebra $K$ is called coreduced if $r_{i}=\infty$ for all $i$, i.e., $K^{*} \cong F[[\bar{t}]]:=F\left[\left[t_{1}, \ldots, t_{d}\right]\right]$. There is a one-to-one correspondence between such bialgebras and formal group laws $\bar{\varphi}=\left(\varphi_{1}, \ldots, \varphi_{d}\right)$, where $\varphi_{i} \in F[[\bar{u}, \bar{v}]]$ have no constant term and satisfy the following axioms: $\bar{\varphi}(\bar{u}, \overline{0})=\bar{u}, \bar{\varphi}(\overline{0}, \bar{v})=\bar{v}$, and $\bar{\varphi}(\bar{\varphi}(\bar{u}, \bar{v}), \bar{w})=\bar{\varphi}(\bar{u}, \bar{\varphi}(\bar{v}, \bar{w}))$ (here $\bar{u}=\left(u_{1}, \ldots, u_{d}\right)$, etc., are formal variables). Namely, given $K$, one identifies $K^{*}$ with $F[[\bar{t}]]$ and sets $\varphi_{i}=\sum_{\alpha, \beta}\left\langle t_{i}, z^{(\alpha)} z^{(\beta)}\right\rangle \bar{u}^{\alpha} \bar{v}^{\beta}$, where $\alpha, \beta$ range over $\mathbb{Z}_{+}^{d}$. Conversely, given a formal group law $\bar{\varphi}$ one defines $K$ as the span of $z^{(\alpha)}, \alpha \in \mathbb{Z}_{+}^{d}$, with coproduct defined by (11) and product $z^{(\alpha)} z^{(\beta)}=$ $\sum_{\gamma} \lambda_{\gamma}^{\alpha, \beta} z^{(\gamma)}$, where $\lambda_{\gamma}^{\alpha, \beta}$ is the coefficient of $\bar{u}^{\alpha} \bar{v}^{\beta}$ in the series $\bar{\varphi}^{\gamma}$ (it is easy to see that the sum defining $z^{(\alpha)} z^{(\beta)}$ is actually finite for any fixed $\left.\alpha, \beta\right)$.

In particular, any linear algebraic group $A$ of dimension $d$ can be "formalized" by choosing a local system of parameters $\left(t_{1}, \ldots, t_{d}\right)$ at the identity and writing the group multiplication in terms of formal power series. This gives rise to a reduced bialgebra $\mathcal{A}$ as outlined above [10, IV, No. 2]. The Lie algebra $P(\mathcal{A})$ is precisely the tangent algebra $\operatorname{Lie}(A)$. For example, the group $G L_{n}(F)$ of dimension $d=n^{2}$ gives the formal group law $\varphi_{(i j)}(\bar{u}, \bar{v})=u_{(i j)}+v_{(i j)}+\sum_{k} u_{(i k)} v_{(k j)}$, where the local system of parameters $\bar{t}$ consists of the entries of the matrix $g-1, g \in G L_{n}(F)$, so the components of $\bar{t}, \bar{u}, \bar{v}$ and $\bar{\varphi}$ are numbered by double subscripts $(i j)$, with $i$ and $j$ running from 1 to $n$. We will denote the corresponding bialgebra by $\mathcal{G}=\mathcal{G}_{n}(F)$.

The natural action of $G L_{n}(F)$ on the space $V=\left\langle e_{1}, \ldots, e_{n}\right\rangle$ corresponds to the following action of $\mathcal{G}: z^{(0)} \cdot e_{j}=e_{j}, z^{\left(\varepsilon_{(i j)}\right)} \cdot e_{j}=e_{i}$, and $z^{(\alpha)} \cdot e_{j}=0$ for all other $\alpha$. The correspondence is in the sense that $h \cdot e_{j}=\sum_{i}\left\langle\delta_{i j}+t_{(i j)}, h\right\rangle e_{i}$ for all $h \in \mathcal{G}$ (where, as before, we identify $\mathcal{G}^{*}$ with $\left.F[[\bar{t}]]\right)$. Note that $P(\mathcal{G})$ acts by the usual matrix multiplication, so we will identify $P(\mathcal{G})$ and $\mathfrak{g l}_{n}(F)$. The action of $\mathcal{G}$ on $V$ has the following universal property. If $H$ is any connected cocommutative bialgebra that acts on $V$, then there exists a unique homomorphism of bialgebras $\eta: H \rightarrow \mathcal{G}$ such that $\eta(h) \cdot x=h \cdot x$ for all $h \in H$ and $x \in V$. Namely, $\eta$ is defined by writing $h \cdot e_{j}=\sum_{i} a_{i j}(h) e_{i}$ where $a_{i j} \in H^{*}$, and then setting $\eta_{\alpha}=\prod_{i, j}\left(a_{i j}-\delta_{i j}\right)^{\alpha(i j)} \in H^{*}$ and $\eta(h)=\sum_{\alpha}\left\langle\eta_{\alpha}, h\right\rangle z^{(\alpha)}$ (the sum is in fact finite for any fixed $h$ because of the connectedness of $H$ ).

If $A \subset G L_{n}(F)$ is a linear algebraic group, then the corresponding bialgebra $\mathcal{A}$ imbeds canonically into $\mathcal{G}_{n}(F)$ [10, IV, No. 3]. A homomorphism $\theta: A \rightarrow B$ of linear algebraic groups induces a homomorphism of bialgebras $\Theta: \mathcal{A} \rightarrow \mathcal{B}$; moreover, $\Theta(\mathcal{A}) \subset \mathcal{B}$ is the subbialgebra corresponding to $\theta(A) \subset B$ [10, IV, No. 3, Proposition 1].

Lemma 3.1. Suppose $A \subset G L(V)$ is a linear algebraic group and $\mathcal{A} \subset \mathcal{G}$ are the corresponding bialgebras. If $v_{0} \in V$ is $A$-invariant, then $v_{0}$ is also $\mathcal{A}$-invariant.

Proof. Pick a basis $\left\{e_{1}, \ldots, e_{n}\right\}$ of $V$ so that $e_{1}=v_{0}$. Then the matrices representing $g \in A$ all have $(1,0, \ldots, 0)^{T}$ as the first column, so $\left\langle t_{(i 1)}, h\right\rangle=0$ for all $h \in \mathcal{A}$. Hence $h \cdot v_{0}=\sum_{i}\left\langle\delta_{i 1}+t_{(i 1)}, h\right\rangle e_{i}=\langle 1, h\rangle e_{1}=\varepsilon_{\mathcal{A}}(h) v_{0}$ for all $h \in \mathcal{A}$, as desired.

Remark 3.2. The converse is also true, but we will not need it here.

We will need one result on subbialgebras of a connected cocommutative bialgebra. If $K$ is such a bialgebra over a field of characteristic zero, then the subbialgebras of $K$ are in one-to-one correspondence with the Lie subalgebras of $P(K)$ via $H \mapsto P(H)$. This is false in positive characteristic. The following lemma allows us, 
under some conditions, to pin down $H \subset K$ by looking at primitive elements. Over a perfect field of characteristic $p>0$, one can define the "shift" (Verschiebung) operator $\mathfrak{V}: K \rightarrow K$ as the dual of the Frobenius map $a \mapsto a^{p}$ on the commutative algebra $K^{*}$. If $\left\{z^{(\alpha)}\right\}$ is a basis of "divided powers" in $K$, then $\mathfrak{V}\left(z^{(\alpha)}\right)=z^{(\alpha / p)}$ if each component of $\alpha$ is divisible by $p$ and $\mathfrak{V}\left(z^{(\alpha)}\right)=0$ otherwise [10, II, $\S 2$, No. 7]. In particular, $K$ is coreduced $\Longleftrightarrow$ the operator $\mathfrak{V}: K \rightarrow K$ is surjective.

Lemma 3.3. Let $K$ be a coreduced connected cocommutative bialgebra and $H_{1} \subset H_{2}$ two subbialgebras. If $\mathfrak{V}^{r}\left(H_{1}\right) \cap P(K)=\mathfrak{V}^{r}\left(H_{2}\right) \cap P(K)$ for all $r \in \mathbb{Z}_{+}$, then $H_{1}=H_{2}$. In particular, if $H_{1}$ is coreduced and $P\left(H_{1}\right)=P\left(H_{2}\right)$, then $H_{1}=H_{2}$.

Proof. The first claim is [10, II, $\S 3$, No. 2, Corollary 1]. As to the second claim, we have $\mathfrak{V}^{r}\left(H_{1}\right) \cap P(K)=H_{1} \cap P(K)=P\left(H_{1}\right)$, because $H_{1}$ is coreduced. Since $P\left(H_{1}\right)=P\left(H_{2}\right)=H_{2} \cap P(K) \supset \mathfrak{V}^{r}\left(H_{2}\right) \cap P(K)$, we conclude that $\mathfrak{V}^{r}\left(H_{1}\right) \cap$ $P(K)=\mathfrak{V}^{r}\left(H_{2}\right) \cap P(K)$ for all $r$ and thus $H_{1}=H_{2}$.

\section{Hopf Algebra aCtions on matrix Lie ALgebras}

Suppose $F$ is an algebraically closed field. Recall that a linear Lie algebra $L \subset M_{n}(F)$ is called algebraic if $L$ is the tangent algebra of some linear algebraic group $A \subset G L_{n}(F)$. Keeping in mind our applications to gradings, we are mostly interested in the case of characteristic $p>0$, but the following result also holds in characteristic zero.

Theorem 4.1. Let $F$ be an algebraically closed field and $L \subset M_{n}(F)$ an algebraic linear Lie algebra such that all derivations of $L$ are inner. Let $R$ be the (unital) associative subalgebra generated by $L$ in $M_{n}(F)$. Suppose a connected cocommutative bialgebra $H$ acts on $L$ so that $L$ is an $H$-module algebra. Then the action of $H$ can be uniquely extended to $R$ so that $R$ is an $H$-module algebra.

Proof. Let $\mathcal{G}$ be the bialgebra corresponding to the algebraic group $G L(L)$, as discussed in Section 3, Then the action of $H$ on $L$ factors through a homomorphism of bialgebras $\eta: H \rightarrow \mathcal{G}$. Let $C \subset \mathcal{G}$ be the largest subcoalgebra such that

$$
k \cdot[x, y]=\sum\left[k_{1} \cdot x, k_{2} \cdot y\right] \text { for all } x, y \in L \text { and } k \in C .
$$

It follows from Remark 2.1 that $C$ is in fact a subbialgebra. Clearly, $\eta(H) \subset C$.

Let $A \subset G L_{n}(F)$ be an algebraic subgroup whose tangent algebra is $L$. Let $\widetilde{A}=\operatorname{Ad} A \subset G L(L)$ and $\widetilde{L}$ be the tangent algebra of $\widetilde{A}$. Then $\operatorname{ad} \subset \widetilde{L}$ and $\widetilde{L} \subset \operatorname{Der} L$. Since all derivations of $L$ are inner by assumption, we have ad $L=\widetilde{L}=$ Der $L$. On the other hand, specializing (2) to primitive elements $k \in C$, we obtain $P(C)=\operatorname{Der} L$. Hence $\widetilde{L}=P(C)$.

Let $\widetilde{\mathcal{A}} \subset \mathcal{G}$ be the subbialgebra corresponding to the algebraic subgroup $\widetilde{A} \subset$ $G L(L)$. Then $\widetilde{\mathcal{A}}$ is coreduced and $P(\widetilde{\mathcal{A}})=\widetilde{L}$. Consider the action of $\widetilde{A}$ on the space $V=\operatorname{Hom}(L \otimes L, L)$ induced by the action on $L$. Let $\theta: \widetilde{A} \rightarrow G L(V)$ and $\Theta: \widetilde{A} \rightarrow \mathcal{G}_{\operatorname{dim} V}(F)$ be the corresponding homomorphisms. Applying Lemma 3.1 to $v_{0}=[,] \in V, \theta(\widetilde{A})$ and $\Theta(\widetilde{\mathcal{A}})$, we see that [, ] is $\Theta(\widetilde{\mathcal{A}})$-invariant, which implies that (2) holds for $k \in \widetilde{\mathcal{A}}$. Hence $\widetilde{\mathcal{A}} \subset C$. Applying Lemma 3.3 with $H_{1}=\widetilde{\mathcal{A}}$ and $H_{2}=C$, we conclude that $\widetilde{\mathcal{A}}=C$.

We have established that $\eta(H) \subset \widetilde{\mathcal{A}}$. Now let $A$ act by conjugation on $M_{n}(F)$. Then $R \subset M_{n}(F)$ is an invariant subspace, and hence we obtain a homomorphism 
$A \rightarrow G L(R)$. Let $\bar{A}$ be the image of this homomorphism and $\bar{L}$ its tangent algebra. Also let $\overline{\mathcal{A}}$ be the subbialgebra of $\mathcal{G}_{\operatorname{dim} R}(F)$ corresponding to $\bar{A} \subset G L(R)$. Since $\bar{A}$ acts by automorphisms of $R$, we can apply Lemma 3.1 again, this time with $V=\operatorname{Hom}(R \otimes R, R)$ and $v_{0} \in V$ the associative multiplication on $R$. We conclude that

$$
k \cdot(x y)=\sum\left(k_{1} \cdot x\right)\left(k_{2} \cdot y\right) \text { for all } x, y \in R \text { and } k \in \overline{\mathcal{A}} .
$$

Lemma 3.1 also implies that $1 \in M_{n}(F)$ is $\overline{\mathcal{A}}$-invariant. Hence $R$ is an $\overline{\mathcal{A}}$-module algebra. The restriction of the action of $\bar{A}$ from $R$ to $L$ gives a homomorphism $\psi: \bar{A} \rightarrow \widetilde{A}$ of algebraic groups and the corresponding homomorphism of bialgebras $\Psi: \overline{\mathcal{A}} \rightarrow \widetilde{\mathcal{A}}$. Clearly, $\psi$ is surjective. Since $L$ generates $R$ as an associative algebra, $\psi$ is also injective. Finally, the differential of $\psi$ is the restriction map $\bar{L} \rightarrow \widetilde{L}$ of the tangent algebras. Since $\bar{L}$ acts by derivations on $R$ and $L$ generates $R$, we see that the restriction map $\bar{L} \rightarrow \widetilde{L}$ is also injective. It follows that $\psi$ is an isomorphism of algebraic groups and thus $\Psi$ is an isomorphism of bialgebras. It remains to define the action of $H$ on $R$ by setting $h \circ x=\Psi^{-1} \eta(h) \cdot x$ for all $x \in R$. Then $R$ is an $H$-module algebra by (3) and by $\overline{\mathcal{A}}$-invariance of $1_{R}$. Also the restriction of the action of $H$ from $R$ to $L$ gives the original action of $H$. The uniqueness of $\circ$ is clear.

Remark 4.2. In geometric language, Theorem 4.1 can be restated as follows. Suppose $F, L$ and $R$ are as in the theorem and an infinitesimal formal group scheme $G$ acts on $L$ so that the Lie bracket is $G$-equivariant. Then the action of $G$ can be uniquely extended to $R$ such that the associative product is $G$-equivariant.

Remark 4.3. Using affine group schemes instead of formal groups in the proof of Theorem 4.1, one can obtain the following result. Let $F, L$ and $R$ be as in the theorem. Let $H$ be a commutative Hopf algebra without nontrivial idempotents. Suppose $L$ is an $H$-comodule algebra via $\rho: L \rightarrow L \otimes H$. Then $\rho$ can be uniquely extended to $\bar{\rho}: R \rightarrow R \otimes H$ so that $R$ is an $H$-comodule algebra. In geometric language, this can be stated as follows: if a connected affine group scheme $G$ acts on $L$ so that the Lie bracket is $G$-equivariant, then the action of $G$ can be uniquely extended to $R$ such that the associative product is $G$-equivariant.

Corollary 4.4. Let $F$ be an arbitrary field of characteristic $p \neq 2$. Let $R=M_{n}(F)$ with $p \nmid n$. Let $H$ be a connected cocommutative bialgebra.

1) If $H$ acts on $R$ such that the Lie algebra $R^{(-)}$is an $H$-module algebra and $1_{R}$ is $H$-invariant, then the associative algebra $R$ is an $H$-module algebra.

2) If the Lie algebra $[R, R]$ is an $H$-module algebra, then extending the action by $h \cdot 1_{R}=\varepsilon(h) 1_{R}$ for all $h \in H$ we turn the associative algebra $R$ into an $H$-module algebra.

Proof. We can assume that $F$ is algebraically closed. It is known that, under the above assumptions on $p$ and $n$, all derivations of $L=\mathfrak{s l}_{n}(F)$ are inner. Applying Theorem 4.1, we obtain 2). Now 1) follows, because $[R, R]$ is an $H$-invariant subspace.

Corollary 4.5. Let $R=M_{n}(F)$, char $F=p>0, p \neq 2$ and $p \nmid n$. Let $G$ be a finite abelian p-group. Suppose $R=\bigoplus_{g \in G} R_{g}$ is a grading on $R^{(-)}$. Then $R=\bigoplus_{g \in G} R_{g}$ is a grading on $R$ if and only if $1 \in R_{1}$. 
Corollary 4.6. Let $F$ be an arbitrary field of characteristic $p \neq 2$. Suppose $R=$ $M_{n}(F)$ and $L$ is either $\mathfrak{s o}_{n}(F)$ or $\mathfrak{s p}_{n}(F)$ ( $n$ even in the latter case). In the case $L=\mathfrak{s o}_{n}(F)$, assume that $n \neq 4$ and, if $p=3, n \neq 3$. Let $H$ be a connected cocommutative bialgebra. Then any action of $H$ on the Lie algebra $L$ can be uniquely extended to an action of $H$ on the associative algebra $R$.

Proof. It is known that, under the above restrictions on $p$ and $n$, all derivations of $L$ are inner; see e.g. [7, Theorems 3.4 and 3.5] or [8].

\section{Gradings on Classical simple Lie algebras}

In this section we apply the results on bialgebra actions to describe all gradings by finite abelian groups on the simple Lie algebras of types $A_{m}$ (except $m=k p+1$ ), $B_{m}, C_{m}$ and $D_{m}$ (except $m=4$ ) over an algebraically closed field $F$ of charactersistic $p \neq 2$.

The gradings on the Lie algebra $L=\mathfrak{s l}_{n}(F)$ over an algebraically closed field $F$ of characteristic 0 have been completely described in [6. Namely, the gradings $L=\bigoplus_{g \in G} L_{g}$ by a finite (abelian) group $G$ are of the following two types:

I : $L_{g}=R_{g}$ for $g \neq 1$ and $L_{1}=R_{1} \cap L$, where $M_{n}(F)=\bigoplus_{g \in G} R_{g}$ is a $G$-grading on $M_{n}(F)$;

II : $L_{g}=\mathcal{K}\left(R_{g}, *\right) \oplus \mathcal{H}\left(R_{g h}, *\right)$ if $g \neq h$ and $L_{h}=\mathcal{K}\left(R_{h}, *\right) \oplus\left(\mathcal{H}\left(R_{1}, *\right) \cap L\right)$ where $M_{n}(F)=\bigoplus_{g \in G} R_{g}$ is a $G$-grading on $M_{n}(F)$, * is an involution that preserves the grading, and $h \in G$ is an element of order 2 . Here $\mathcal{H}(R, *)$ and $\mathcal{K}(R, *)$ stand for the subspaces of symmetric and antisymmetric elements, respectively.

The proof of this result in [6] is based on the following key ideas. First, in this case the gradings by a finite abelian group $G$ are equivalent to the actions of $\widehat{G}$ by automorphisms. Second, any inner automorphism of $\mathfrak{s l}_{n}(F)$ uniquely extends to an automorphism of $M_{n}(F)$ and any outer automorphism to the negative of an antiautomorphism of $M_{n}(F)$. Third, the antiautomorphisms of $M_{n}(F)$ that may arise here can be "corrected" by slightly changing the $\widehat{G}$-action so they become automorphisms (see Proposition 5.3 below). Finally, the original grading on $\mathfrak{s l}_{n}(F)$ can be recovered from the grading associated to the modified action on $M_{n}(F)$ by using an "exchange formula" (see Lemma 5.4 below).

We will extend the above approach, by using our Corollary 4.4, to describe the gradings on $\mathfrak{s l}_{n}(F)$ where $F$ is of positive characteristic $p \neq 2$ not dividing $n$. It turns out that in this case the answer is the same as in characteristic zero:

Theorem 5.1. Let $L=\mathfrak{s l}_{n}(F)$, where $F$ is an algebraically closed field of characteristic $p, p \neq 2$ and $p \nmid n$. Let $G$ be a finite abelian group. Then any $G$-grading on $L$ is either of type $I$ or of type II above. Moreover, if $G$ is a p-group, then any $G$-grading on $L$ is of type $I$, i.e., the restriction of an elementary $G$-grading of $M_{n}(F)$.

Proof. As discussed in Section 2, the gradings by $G$ are equivalent to the actions of the Hopf algebra $K=(F G)^{*}$. We write $G=G_{0} \times G_{1}$, where $G_{0}$ is of order not divisible by $p$ and $G_{1}$ is a $p$-group. Then $K=K_{0} \otimes K_{1}$, where $K_{0}=\left(F G_{0}\right)^{*}=F \widehat{G_{0}}$ and $K_{1}=\left(F G_{1}\right)^{*}$. As in the case of characteristic zero, the action of $\widehat{G_{0}}$ on $\mathfrak{s l}_{n}(F)$ can be extended to $M_{n}(F)$, thanks to results of Blau and Martindale, summarized as follows: 
Theorem 5.2 ([9, Theorem 6.1]). Let $S=M_{m}(E), R=M_{n}(F), n>1$, and let $E$ and $F$ be fields with isomorphism $\gamma: F \rightarrow E$. Assume that char $E \neq 2$, and $m \neq 3$ if char $E=3$. Suppose there is a $\gamma$-semilinear Lie isomorphism $\alpha: \overline{[R, R]} \rightarrow \overline{[S, S]}$, where $\overline{[R, R]}=[R, R] /[R, R] \cap F$ and $\overline{[S, S]}=[S, S] /[S, S] \cap E$. Then $n=m$ and there exists a $\gamma$-semilinear map $\sigma: R \rightarrow S$ such that $\sigma$ is either an isomorphism or the negative of an antiisomorphism and such that $\overline{x^{\alpha}}=\bar{x}^{\alpha}$ for all $x \in[R, R]$.

In our case, $E=F, \gamma=\mathrm{id}, R=S$, and $\operatorname{char} F \nmid n$, so $\overline{[R, R]}=\mathfrak{s l}_{n}(F)$. Thus we can extend (uniquely) the action of $\widehat{G_{0}}$ on $\mathfrak{s l}_{n}(F)$ to $R=M_{n}(F)$ and obtain a homomorphism $f: \widehat{G_{0}} \rightarrow G L(R)$ whose image consists of automorphisms and, possibly, the negatives of antiautomorphisms of $R$, which are all automorphisms of the Lie algebra $R^{(-)}$.

We also extend the $K_{1}$-action on $\mathfrak{s l}_{n}(F)$ to an action on $R^{(-)}$by declaring that the identity matrix is $K_{1}$-invariant. Then by Corollary 4.4 this action turns the associative algebra $R$ into a $K_{1}$-module algebra.

The extended action of $K_{0} \otimes K_{1}$ on $R^{(-)}$corresponds to a Lie grading on $R$, $R=\bigoplus_{g \in G} R_{g}$, which restricts to the original $G$-grading on $\mathfrak{s l}_{n}(F)$.

Now set $\Lambda=f^{-1}\left(\right.$ Aut $R$ ). This is a subgroup in $\widehat{G_{0}}$ of index at most 2 that acts by automorphisms on $R$. Set $H=\Lambda^{\perp}$ in $G_{0}$. Then $H=\langle h\rangle$, where $h \in G_{0}$ is of order at most 2. Set $\bar{K}=F \Lambda \otimes K_{1}$. Then $\bar{K}=(F \bar{G})^{*}$, where $\bar{G}=G / H$. By construction, $R$ is a $\bar{K}$-module algebra. This means that the corresponding factor-grading by $\bar{G}, R=\bigoplus_{\bar{g} \in \bar{G}} R_{\bar{g}}$ where $R_{\bar{g}}=\bigoplus_{g \in \bar{g}} R_{g}$, is a grading of $R$ as an associative algebra.

If $\Lambda=\widehat{G_{0}}$, then we are done: we have a type I grading on $\mathfrak{s l}_{n}(F)$. Otherwise $\widehat{G_{0}}$ is generated over $\Lambda$ by an element $\chi$ such that $f(\chi)=-\varphi$, where $\varphi$ is an antiautomorphism of $R$. Since $\chi$ commutes with $\bar{K}, \varphi$ preserves the $\bar{G}$-grading on $R$. Moreover, $\chi^{2} \in \Lambda$ implies that $\varphi^{2}$ acts trivially on the identity component of the $\bar{G}$-grading. Thus we can apply (for $\bar{G}$ ) the following result of [6], whose proof does not require any assumptions about the characteristic:

Proposition 5.3 ([6. Proposition 6.4]). Let $R=M_{n}(F)$ be graded by a finite abelian group $G$. Let $\varphi$ be an antiautomorphism of $R$ that preserves the grading and acts as an involution on the identity component. Then there exists an automorphism $\psi$ of $R$ that also preserves the grading such that $\varphi$ commutes with $\psi$ and $\varphi^{2}=\psi^{2}$.

Now we can define a new $K$-action on $R$ by making $\chi$ act as $\psi$ (instead of $-\varphi$ ) and $\bar{K}$ as before. By construction, $R$ is a $K$-module algebra with respect to this new action, so the corresponding grading $R=\bigoplus_{g \in G} \widetilde{R}_{g}$ is a grading of $R$ as an associative algebra. Moreover, $*=\psi^{-1} \varphi$ is an involution on $R$ that preserves both gradings $R=\bigoplus_{g \in G} R_{g}$ and $R=\bigoplus_{g \in G} \widetilde{R}_{g}$.

We need one more result, which generalizes the dual version of the so-called "Exchange Theorem"; see for example 2. As opposed to the earlier versions, the result in the present formulation has no restrictions on the characteristic of the base field; its simple proof is left to the reader.

Suppose $R$ is a vector space, $G$ a group, and $R=\bigoplus_{g \in G} R_{g}$ and $R=\bigoplus_{g \in G} \widetilde{R}_{g}$ are two $G$-gradings. We will call these gradings compatible if for all $g \in G, \widetilde{R}_{g}=$ $\bigoplus_{x \in G}\left(R_{x} \cap \widetilde{R}_{g}\right)$, or, equivalently, $R_{g}=\bigoplus_{x \in G}\left(\widetilde{R}_{x} \cap R_{g}\right)$. 
Lemma 5.4. Let $R$ be a vector space with two compatible gradings $R=\bigoplus_{g \in G} R_{g}$ and $R=\bigoplus_{g \in G} \widetilde{R}_{g}$. Suppose $H \triangleleft G$ is such that the two factor-gradings by $G / H$ coincide. Set $R^{h}=\bigoplus_{g \in G}\left(\widetilde{R}_{g} \cap R_{g h}\right)$. Then

$$
R_{g}=\bigoplus_{h \in H}\left(\widetilde{R}_{g h^{-1}} \cap R^{h}\right) .
$$

Moreover, if $R$ is a (nonassociative) algebra equipped with two such gradings and $H \subset Z(G)$, then $R=\bigoplus_{h \in H} R^{h}$ is an algebra grading.

We apply Lemma 5.4 in order to express $R_{g}$ through $\widetilde{R}_{g}$ as follows. In our case $R^{1}=\bigoplus_{g \in G}\left(\widetilde{R}_{g} \cap R_{g}\right)=\bigoplus_{g \in G} \mathcal{K}\left(\widetilde{R}_{g}, *\right)=\mathcal{K}(R, *), R^{h}=\bigoplus_{g \in G}\left(\widetilde{R}_{g} \cap R_{g h}\right)=$ $\bigoplus_{g \in G} \mathcal{H}\left(\widetilde{R}_{g}, *\right)=\mathcal{H}(R, *)$. Therefore,

$$
R_{g}=\left(\widetilde{R}_{g} \cap R^{1}\right) \oplus\left(\widetilde{R}_{g h} \cap R^{h}\right)=\mathcal{K}\left(\widetilde{R}_{g}, *\right) \oplus \mathcal{H}\left(\widetilde{R}_{g h}, *\right) .
$$

Restricting $R_{g}$ to $\mathfrak{s l}_{n}(F)$, we see that we have a grading of type II.

This completes the proof of Theorem 5.1

A similar but simpler argument (no antiautomorphisms involved), using our Corollary 4.6 and also [7, Corollary 1.7] to extend automorphisms, yields the second main result of the paper.

Theorem 5.5. Let $L$ be one of $\mathfrak{s o}_{n}(F), n \geq 5, n \neq 8$, and $\mathfrak{s p}_{n}(F), n \geq 6, n$ even, where $F$ is an algebraically closed field of characterisitc $p \neq 2$. Let $G$ be a finite abelian group. Then any $G$-grading on $L$ is the restriction of a $G$-grading of $M_{n}(F)$. Moreover, if $G$ is a p-group, then any $G$-grading on $L$ is the restriction of an elementary $G$-grading of $M_{n}(F)$.

Since the involutions on graded matrix algebras are described in [4, Theorems 5.1 and 5.5 give a complete description of gradings on all classical simple Lie algebras over algebraically closed fields of characteristic $p \geq 3$, with the exception of the simple Lie algebras of the form $\mathfrak{s o}_{8}(F)$ and $\mathfrak{p s l}_{n}(F)=\mathfrak{s l}_{n}(F) / F$, where $p \mid n$.

\section{ACKNowledgments}

We would like to thank the referee of the paper, whose valuable suggestion to use group schemes allowed us to considerably streamline the proofs and led us to much stronger results. We also thank Professor Alexander Premet for his important advice about simple Lie algebras in characteristic $p$.

\section{REFERENCES}

[1] Bahturin, Y.; Goze, M. $\mathbb{Z}_{2} \times \mathbb{Z}_{2}$-symmetric spaces, Pacific J. Math., 236 (2008), 1-21.

[2] Bahturin, Y.; Giambruno, A. Group gradings on associative algebras with involution, Canad. Math. Bull., 51 (2008), 182-194.

[3] Bahturin, Y.; Shestakov, I.; Zaicev, M. Gradings on simple Jordan and Lie algebras, J. Algebra, 283 (2005), 849-868. MR2111225 (2005i:17038)

[4] Bahturin, Y.; Zaicev, M. Involutions on graded matrix algebras, J. Algebra, 315 (2007), 527-540. MR2351876

[5] Bahturin, Y.; Zaicev, M. Graded algebras and graded identities, Polynomial identities and combinatorial methods (Pantelleria, 2001), 101-139, Lecture Notes in Pure and Appl. Math., 235, Dekker, New York, 2003. MR2021796 (2005a:16059)

[6] Bahturin, Y.; Zaicev, M. Gradings on simple Lie algebras of type "A", J. Lie Theory, 16 (2006), 719-742. MR2270657 (2007i:17037) 
[7] Beidar, K. I.; Brešar, M.; Chebotar, M. A.; Martindale, W. S., 3rd. On Herstein's Lie map conjectures. III, J. Algebra, 249 (2002), no. 1, 59-94. MR1887985 (2003c:16042)

[8] Benkart, G.; Gregory, T.; Premet, A. The recognition theorem for graded Lie algebras in prime characteristic, arXiv:math.RA/0508373 v2 (29 Sep 2005).

[9] Blau, P. S.; Martindale, W. S., 3rd. Lie isomorphisms in *-prime GPI rings with involution, Taiwanese J. Math., 4 (2000), 215-252. MR1757403 (2001i:16061)

[10] Dieudonné, J. Introduction to the theory of formal groups. Pure and Applied Mathematics, 20, Marcel Dekker, Inc., New York, 1973. MR0332802 (48:11128)

[11] Jantzen, J. C. Representations of algebraic groups. Second edition. Mathematical Surveys and Monographs, 107, American Math. Soc., Providence, RI, 2003. MR2015057 (2004h:20061)

[12] Kac, V. Graded algebras and symmetric spaces. Funct. Anal. Pril., 2 (1968), 93-94. MR0231944 (38:270)

[13] Kac, V. Infinite dimensional Lie algebras, second edition, Cambridge University Press, 1985. MR $823672(87 \mathrm{c}: 17023)$

[14] Montgomery, S. Hopf algebras and their actions on rings, CBMS Regional Conference Series in Mathematics, 82, American Math. Soc., Providence, RI, 1993. MR 1243637 (94i:16019)

[15] Zelmanov, E. Lie algebras with a finite grading. Engl. Transl., Math. USSR-Sb., 52 (1985), 347-385. MR0752226

Department of Mathematics and Statistics, Memorial University of Newfoundland, St. John's, NL, A1C5S7, CANADA

E-mail address: yuri@math.mun.ca

Department of Mathematics and Statistics, Memorial University of Newfoundland, St. John's, NL, A1C5S7, CANADA

E-mail address: mikhail@math.mun.ca

Department of Mathematics, University of Southern California, 3620 South Vermont Avenue, KAP 108, Los Angeles, California 90089-2532

E-mail address: smontgom@math.usc.edu 